\title{
Medically Integrated Fitness Program Review to Improve Health and Functional Outcomes
}

\author{
Michael M.Haniff, MD, PhD(c) ${ }^{2}$, Christopher R.Sutton, MS, ACSM-RCEP, EIM ${ }^{1}$, James J.L.Mateka, MD, \\ PhD(c) ${ }^{2}$, Peggy Mc Donagh Bravo, RN, BSN, MA', Wesley R. Waggener, PhD, CSCS ${ }^{1}$ and Nathaniel S. \\ Jones, MD, CAQ-SM ${ }^{3}$ \\ ${ }^{1}$ Power Wellness Management, USA \\ ${ }^{2} \mathrm{HM}$ Healthcare Partners, USA \\ ${ }^{3}$ Loyola University Medical Center, USA

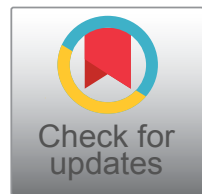

*Corresponding author: Michael M. Haniff, MD, PhD (c), HM Healthcare Partners, Ft.Lauderdale, FL33319, USA, Tel: 312 985-9300

\begin{abstract}
Purpose: Exercise prescription may be a useful tool in the management and prevention of chronic disease among the adult population. There is a dearth of structured exercise prescription programs for improving quality of life, physical function, and overall health. The purpose of this investigationwas to evaluate the effects of NextSteps Fitness Programs ${ }^{\mathrm{TM}}$ on symptoms and health outcomes among adults with one or more chronic conditions.

Methods: This study was a retrospective examination of NextSteps Fitness Program ${ }^{\text {TM }}$ participants $(n=7,971)$ between January 2014 to December 2017. Paired t-tests were used to assess percent change between preassessment to post-assessment.

Results: Nearly three quarters ( $n=5,753 ; 72 \%$ ) of participants completed the 8-week program. Change from pre-assessment to post-assessment within the six fitness pathways using paired t-tests revealed mostly significant improvements (adjusted $P<0.01$ ) in BMI, diastolic blood pressure, V02max, number of crunches, isometric bicep strength and mental and physical compositescores.

Conclusion: These findings suggest this structured and personalized fitness program is effective and holds much promise to improve general health and slow disease progression among older adults with one or more chronic conditions if prescribed by the managing care provider.
\end{abstract}

\section{Keywords}

Prescriptive fitness, Exercise training, Exercise prescription, Health outcomes, Chronicdisease, Adult

\section{Introduction}

Chronic conditions and comorbidities such as cardiovascular disease (CVD), diabetes, cancer, and chronic lower respiratory disease are among the leading causes of mortality in the United States. According to The Centers for Disease Control and Prevention (CDC), approximately $60 \%$ of adults in the United States suffer from at least one chronic condition, and over $42 \%$ have two or more [1]. Furthermore, the estimates of chronic and mental health conditions account for $90 \%$ of the nation's $\$ 3.3$ trillion health care expenditures each year. By 2050, the population over the age of 65 is projected to reach approximately 84 million, almost double the amount in 2012 [1]. Due to this unprecedented rise in older adults, and the concomitant rise in chronic disease, as well as the associated inactivity in this population, the need to incorporate efficient and effective translational evidence-based physical activity interventions remains vital [2].

Physical activity in the form of exercise has been well documented in the literature. It has proven to be effective in the prevention, onset, and treatment for most chronic conditions [3-5] such as cardiovascular disease (CVD) $[6,7]$, chronic obstructive pulmonary disease (COPD) [8], depression [9], diabetes [10], osteoarthritis [11], osteoporosis [12,13], and cancers such as

Citation: Haniff MM, Sutton CR, Mateka JJL, Bravo PD, Waggener WR (2021) Medically Integrated Fitness Program Review to Improve Health and Functional Outcomes. Int J Sports Exerc Med 7:188. doi. org/10.23937/2469-5718/1510188

Accepted: April 19, 2021; Published: April 21, 2021

Copyright: (C) 2021 Haniff MM, et al. This is an open-access article distributed under the terms of the Creative Commons Attribution License, which permits unrestricted use, distribution, and reproduction in any medium, provided the original author and source are credited. 
colon [14], breast [15], endometrial [16], and moderate effects against prostate [17], lung and ovarian cancers. The 2018 Physical Activity Guidelines for Americans recommend that adults engage in 150 minutes of moderate-intensity physical activity and/or two or more weekly muscle-strengthening activities to gain health benefits [18]. Evidence also shows that there is a positive association between regular exercise and improved quality of life in populations with chronic disease [19].

Physical activity in the form of exercise prescription has been established as a low-cost intervention in chronic illness to improve overall health, quality of life, and physical function in older adults [20-22]. The CDC, The American College of Sports Medicine (ACSM), and the American Heart Association have recommended that health care practitioners provide counseling and referrals of their patients to participate in physical activity programs designed for specialized populations $[13,18,23]$. Research suggests that if recommended by the patient's care provider or physician, exercise is considered valuable by the patient [24], yet less than onethird of providers recommend exercise counseling or education to their patient population [25]. Improved exercise prescription could reduce complications and functional declines seen in the aging population and provide patients with optimized treatment. Recommending an exercise prescription plan to patients with one or more chronic conditions can help maximize health benefits and bend the cost-curve among this significantly growing proportion of our population.

To date, there exists a lack of evidence-based, effective, and structured physical activity curriculum within community settings that ensure the dissemination of proper physical activities among adults with one or more chronic conditions. To bridge the gap, Next Steps Fitness Programs ${ }^{T M}$ was developed as a medically integrated, multicomponent exercise and behavioral change intervention designed to deliver individualized fitness protocols for those transitioning from or managing one or more chronic conditions. This investigation aimed to evaluate the effects of NextSteps Fitness Programs $^{T M}$ on symptoms and health outcomes among older adults with one or more chronic conditions, including heart disease, cancer, diabetes, respiratory, obesity, and orthopedics.

\section{Methods}

A pre-assessment-post-assessment study design was used to examine the benefits of NextSteps Fitness Programs $^{T M}$ among participants with one or more chronic conditions. This retrospective evaluation was approved under Independent Review Board (Sterling \#6260) exemption. All identifying information was removed before any research activities.

\section{Sample}

The sample was a cohort of NextSteps Fitness Pro- gram $^{T M}$ participants who were medically cleared to participate in the fitness protocol by their referring physician or other health care provider into one of the various programs. NextSteps Fitness Program ${ }^{T M}$ participants completed an 8-week chronic disease pathway (cardiac fitness, cancer fitness, diabetes fitness, pulmonary fitness, weight management, and orthopedic fitness) at one of the 21 fitness facilities located throughout the United States from January 2014 to December 2017 (n $=7,971$ ). Successful program completion was defined as the fulfillment of program requirements, including preand post-health assessments.

\section{Clinical measures}

A centrally managed repository, PowerLink ${ }^{\mathrm{TM}}$ (Lombard, IL) was used to collect informed consent, health history questionnaire, risk stratification data, demographics, and pre- and post-assessment health measures. Age and sex were self-reported. The health measures included fitness-specialists measured anthropometric data at baseline and conclusion of the 8-week NextSteps program pathway. The measures included body fat percentage, body mass index, and weight in kilograms using a calibrated Tanita ${ }^{\circledR}$ BF-350 (Arlington Heights, IL) scale, height in inches using Seca ${ }^{\circledR}$ stadiometer (Chino, CA), estimated maximum oxygen consumption $\left(\mathrm{VO}_{2}\right.$ max) using Polar BodyAge ${ }^{\circledR}$ OwnIndex ${ }^{\circledR}$ and Polar ${ }^{\circledR} \mathrm{H} 2$ Bluetooth Heart Rate Chest Transmitter (Bethpage, NY), and blood pressure using MooreBrand ${ }^{\circledR}$ Standard Aneroid Sphygmomanometer and MooreBrand ${ }^{\circledR}$ Sprague-Rappaport Stethoscope (Farmington, CT). Additional measures collected included health-related quality of life using the 36-item, short-form health survey questionnaire (SF-36) for physical composite score (PCS) and the mental composite score (MCS). BMI was calculated using weight in kilograms divided by the square of the converted height in meters $(\mathrm{kg} / \mathrm{m} 2)$. Measures of strength and endurance were assessed using Polar BodyAge ${ }^{\circledR}$ Four Load Cell Platform (Bethpage, NY) and the total number of abdominal crunches. Data was recorded by a certified fitness specialist into the Polar BodyAge ${ }^{\circledR}$ system software.

\section{Statistical analysis}

Descriptive statistics were used to report demographics by each program. Pre- and post-assessment evaluation measurements were summarized across all participants using means and standard deviations, stratified by NextSteps Fitness Program ${ }^{T M}$ pathway. The univariate changes were analyzed using a paired t-test of the participant-matched pre-assessment (baseline), and post-assessment (end of the 8-week program) measures at an adjusted significance level of 0.05 . The percent change between the pre- post-assessment measures were calculated of the measurements including BMI, diastolic blood pressure, estimated $\mathrm{VO}_{2} \mathrm{~m}$ ax, number of crunches, arm strength (in pounds) and self-reported values of the SF-36 physical and mental composite scores, by dividing the overall change across 
participants by the mean of the respected pre-assessment score. All analyses were conducted using SPSS 23 (Chicago, IL).

\section{Results}

\section{Participant characteristics}

Enrollment into the 8-week Next Steps Fitness Programs $^{T M}$ began in 2014 and is currently ongoing. The data presented in this evaluation pertain to adult participants from January 2015 to December 2017. A total of 7,971 participants were enrolled in one of the six Next Steps Fitness Program ${ }^{T M}$ pathways with a mean age of 60.61 (SD $=12.67)$ years. The majority of participants were female $(n=5,345,67 \%)$. A total of $5,753(72 \%)$ met the study criteria, defined as having completed a preand post-assessment within one of the six evaluated program pathways (cardiac fitness, cancer fitness, diabetes fitness, orthopedic fitness, pulmonary fitness, and weight management). Change from pre-assessment to post-assessment within the six program pathways using paired t-tests revealed mostly significant improvements (adjusted $P<0.01$ ) in $\mathrm{BMI}$, diastolic blood pressure, VO2max, number of crunches, isometric bicep strength and in the mental and physical composite scores using SF36 (Table 1). Overall, NextSteps Fitness Program ${ }^{\text {TM }}$ participants in the six program pathways experienced the greatest improvements in the total number of crunches

Table 1: Results of t-test analysis.

\begin{tabular}{|c|c|c|c|c|c|}
\hline Program & $\mathbf{N}$ & Pretest (Mean) & Posttest (Mean) & Change (\%) & Adjusted p-Value \\
\hline \multicolumn{6}{|l|}{ Weight Management } \\
\hline BMI & 1122 & $37.45 \pm 8.10$ & $36.70 \pm 7.91$ & $-0.74(-2 \%)$ & $<0.01$ \\
\hline Diastolic BP & 1123 & $78.32 \pm 9.05$ & $77.08 \pm 8.55$ & $-1.23(-1 \%)$ & $<0.01$ \\
\hline $\mathrm{VO}_{2}$ & 799 & $24.38 \pm 5.48$ & $25.64 \pm 5.72$ & $1.266(4 \%)$ & $<0.01$ \\
\hline Crunches & 677 & $32.95 \pm 15.77$ & $43.83 \pm 15.96$ & $10.87(24 \%)$ & $<0.01$ \\
\hline Biceps & 796 & $56.41 \pm 27.10$ & $64.35 \pm 31.08$ & $7.942(12 \%)$ & $<0.01$ \\
\hline SF-36 Mental Composite Score & 981 & $50.38 \pm 10.53$ & $53.85 \pm 8.70$ & $3.465(6 \%)$ & $<0.01$ \\
\hline SF-36 Physical Composite Score & 983 & $45.30 \pm 9.61$ & $48.96 \pm 8.23$ & $3.666(7 \%)$ & $<0.01$ \\
\hline \multicolumn{6}{|l|}{ Diabetes Fitness } \\
\hline BMI & 444 & $34.36 \pm 7.32$ & $34.14 \pm 7.21$ & $-0.22(-0 \%)$ & $<0.01$ \\
\hline Diastolic BP & 442 & $76.66 \pm 9.08$ & $75.00 \pm 8.56$ & $-1.65(-2 \%)$ & $<0.01$ \\
\hline $\mathrm{VO}_{2}$ & 297 & $24.33 \pm 6.44$ & $25.62 \pm 6.09$ & $1.291(5 . \%)$ & $<0.01$ \\
\hline Crunches & 228 & $30.89 \pm 15.37$ & $40.72 \pm 15.24$ & $9.830(24 \%)$ & $<0.01$ \\
\hline Biceps & 286 & $54.74 \pm 21.84$ & $60.80 \pm 23.15$ & $6.058(9 . \%)$ & $<0.01$ \\
\hline SF-36 Mental Composite Score & 406 & $50.55 \pm 10.57$ & $54.16 \pm 8.23$ & $3.613(6 . \%)$ & $<0.01$ \\
\hline SF-36 Physical Composite Score & 406 & $43.68 \pm 9.34$ & $47.40 \pm 8.53$ & $3.724(7 . \%)$ & $<0.01$ \\
\hline \multicolumn{6}{|l|}{ Cardiac Fitness } \\
\hline BMI & 771 & $30.54 \pm 6.50$ & $30.43 \pm 6.40$ & $-0.10(-0 \%)$ & 0.01 \\
\hline Diastolic BP & 768 & $73.87 \pm 10.06$ & $73.52 \pm 9.55$ & $-0.34(-0 \%)$ & 0.29 \\
\hline $\mathrm{VO}_{2}$ & 458 & $25.47 \pm 7.23$ & $26.52 \pm 7.20$ & $1.043(3 . \%)$ & $<0.01$ \\
\hline Crunches & 311 & $29.04 \pm 15.55$ & $39.02 \pm 15.67$ & $9.974(25 \%)$ & $<0.01$ \\
\hline Biceps & 435 & $51.72 \pm 23.33$ & $58.23 \pm 26.41$ & $6.508(11 \%)$ & $<0.01$ \\
\hline SF-36 Mental Composite Score & 684 & $51.82 \pm 9.57$ & $54.34 \pm 8.31$ & $2.524(4 . \%)$ & $<0.01$ \\
\hline SF-36 Physical Composite Score & 685 & $43.38 \pm 9.72$ & $46.26 \pm 9.36$ & $2.881(6 . \%)$ & $<0.01$ \\
\hline \multicolumn{6}{|l|}{ Pulmonary Fitness } \\
\hline BMI & 177 & $30.72 \pm 8.08$ & $30.80 \pm 8.05$ & $0.078(0 . \%)$ & 0.59 \\
\hline Diastolic BP & 176 & $75.09 \pm 10.72$ & $72.13 \pm 8.50$ & $-2.96(-4 \%)$ & $<0.01$ \\
\hline $\mathrm{VO}_{2}$ & 93 & $23.13 \pm 8.29$ & $25.13 \pm 7.55$ & $1.992(7 . \%)$ & $<0.01$ \\
\hline Crunches & 57 & $22.77 \pm 13.75$ & $31.24 \pm 15.58$ & $8.473(27 \%)$ & $<0.01$ \\
\hline Biceps & 93 & $43.57 \pm 18.81$ & $47.85 \pm 22.57$ & $4.287(8 . \%)$ & 0.00 \\
\hline SF-36 Mental Composite Score & 151 & $50.52 \pm 11.26$ & $54.06 \pm 8.99$ & $3.543(6 . \%)$ & $<0.01$ \\
\hline SF-36 Physical Composite Score & 151 & $36.26 \pm 9.07$ & $39.79 \pm 9.43$ & $3.529(8 . \%)$ & $<0.01$ \\
\hline \multicolumn{6}{|l|}{ Orthopedic Fitness } \\
\hline BMI & 1502 & $31.23 \pm 7.16$ & $31.06 \pm 7.05$ & $-0.17(-0 \%)$ & $<0.01$ \\
\hline Diastolic BP & 1504 & $76.05 \pm 9.67$ & $74.76 \pm 9.05$ & $-1.28(-1 \%)$ & $<0.01$ \\
\hline
\end{tabular}




\begin{tabular}{|l|l|l|l|l|l|}
\hline $\mathrm{VO}_{2}$ & 907 & $24.28 \pm 7.08$ & $25.57 \pm 7.06$ & $1.286(5 . \%)$ & $<0.01$ \\
\hline Crunches & 618 & $31.00 \pm 15.36$ & $42.76 \pm 16.18$ & $11.75(27 \%)$ & $<0.01$ \\
\hline Biceps & 917 & $47.01 \pm 25.38$ & $52.64 \pm 25.41$ & $5.630(10 \%)$ & $<0.01$ \\
\hline SF-36 Mental Composite Score & 1322 & $51.76 \pm 10.68$ & $54.22 \pm 9.19$ & $2.462(4 . \%)$ & $<0.01$ \\
\hline SF-36 Physical Composite Score & 1322 & $38.82 \pm 9.15$ & $43.65 \pm 9.15$ & $4.828(11 \%)$ & $<0.01$ \\
\hline Cancer Fitness & & & & & 0.22 \\
\hline BMI & 299 & $30.20 \pm 6.80$ & $30.07 \pm 6.93$ & $-0.13(-0 \%)$ & 0.24 \\
\hline Diastolic BP & 294 & $74.65 \pm 8.71$ & $74.04 \pm 8.60$ & $-0.61(-0 \%)$ & 0.00 \\
\hline V0 & 192 & $24.68 \pm 6.37$ & $25.79 \pm 6.01$ & $1.106(4 . \%)$ & $<0.01$ \\
\hline Crunches & 138 & $28.00 \pm 13.43$ & $38.44 \pm 16.37$ & $10.43(27 \%)$ & $<$ \\
\hline Biceps & 178 & $44.68 \pm 22.65$ & $48.10 \pm 16.56$ & $3.420(7 . \%)$ & 0.02 \\
\hline SF-36 Mental Composite Score & 259 & $47.91 \pm 11.07$ & $52.49 \pm 8.88$ & $4.579(8 . \%)$ & $<0.01$ \\
\hline SF-36 Physical Composite Score & 259 & $41.14 \pm 9.18$ & $44.85 \pm 9.08$ & $3.710(8 . \%)$ & $<0.01$ \\
\hline
\end{tabular}

and the physical and mental composite scores, indicating an increase in muscular endurance and perceived quality of life.

\section{Body Mass Index}

Most participants experienced no change in BMI with the exception of participants within the weight management pathway, who experienced a significant decrease in $\mathrm{BMI}$ between pre-assessment $(\underline{X}=37.45, \mathrm{SE}=0.24)$ and post-assessment $(\underline{X}=36.71, \mathrm{SE}=0.24$, mean difference $=-0.75$, percent change $=-2.03 \%, t(1,121)$ $=-12.51$, adjusted $P<0.001$; Table 1$)$. Changes in BMI were not observed for the other 5 pathways (cardiac fitness, cancer fitness, diabetes fitness, pulmonary fitness, and orthopedic fitness).

\section{Diastolic blood pressure}

Approximately three-quarters of participants (75.3\%; $\mathrm{n}=3,245)$ experienced a decrease and nearly one-quarter $(24.7 \% ; n=1,070)$ experienced no change in diastolic blood pressure. Decreases were observed within weight management (pre-assessment $\underline{X}=78.32, \mathrm{SE}=0.27$, post-assessment $\underline{X}=77.09, \mathrm{SE}=0.26$, mean difference $=-1.236$, percent change $=-1 \%, t(1,122)=-4.645$, ad justed $P<0.001$ ), diabetes fitness (pre-assessment $\underline{X}=$ 76.66, $\mathrm{SE}=0.43$, post-assessment $X=75.01, \mathrm{SE}=0.41$, mean difference $=-1.65$, percent change $=-2 \%, t(441)=$ -3.90, adjusted $P<.001$ ), pulmonary fitness (pre-assessment $\underline{X}=75.10$, SE $=0.81$, post-assessment $\underline{X}=72.14$, $\mathrm{SE}=0.64$, mean difference $=-2.96$, percent change $=$ $-4 \%, t(175)=-4.58$, adjusted $P<0.001)$, and orthopedic fitness pathways (pre-assessment $\underline{X}=76.05, \mathrm{SE}=0.25$, post-assessment $\underline{X}=74.77, \mathrm{SE}=0.23$, mean difference $=-1.28$, percent change $=-1 \%, t(1,503)=-5.64$, adjust ed $P<0.001$; Table 1). Participants within the cardiac fitness and cancer fitness pathways exhibited no significant change in diastolic blood pressure.

\section{$\mathrm{VO}_{2} \max$}

Participants in each pathway $(n=2,746)$ experienced an increase in aerobic capacity. The greatest change was observed within the pulmonary fitness pathway (pre-assessment $X=23.14, \mathrm{SE}=0.86$, post-assessment $X=25.13, \mathrm{SE}=0.78$, mean difference $=1.99$, percent change $=7.0 \%, t(92)=-4.58$, adjusted $P<0.001)$. Both the diabetes fitness (pre-assessment $\underline{X}=24.33, \mathrm{SE}=$ 0.37 , post-assessment $\underline{X}=25.63$, $\mathrm{SE}=0.35$, mean difference $=1.29$, percent change $=5.4 \%, t(296)=-1.73$, adjusted $P<0.001$ ) and orthopedic fitness (pre-assessment $X=24.28, \mathrm{SE}=0.24$, post-assessment $X=25.57$, $\mathrm{SE}=0.23$, mean difference $=1.29$, percent change $=$ $5.0 \%, t(906)=10.34$, adjusted $P<0.001$; Table 1) pathways experienced the second greatest change in aerobic capacity.

\section{Crunches}

Participants in each pathway greatly improved in muscle endurance as measured by the total number of abdominal crunches $(n=2,029)$. However, participants within the orthopedic fitness pathway exhibited the greatest improvement in the number of abdominal crunches performed from pre-assessment $(\underline{X}=31.00$, $\mathrm{SE}=0.62)$ to post-assessment $(\underline{X}=42.76, \mathrm{SE}=0.65$, mean difference $=11.76$, percent change $=27.4 \%, t(617)$ $=25.68$, adjusted $P<0.001$; Table 1 ).

\section{Bicep curls}

We observed an improvement in the isometric biceps curl strength test amongst all Next Steps Fitness Program $^{T M}$ participants $(n=2,705)$. The greatest improvement was observed within the weight management pathway (pre-assessment $X=56.41$, $\mathrm{SE}=0.96$, post-assessment $\underline{X}=64.36, \mathrm{SE}=1.10$, mean difference $=7.94$, percent change $=12 \%, t(795)=13.79$, adjusted $P<0.001$; Table 1 ).

\section{SF-36 Physical \& mental composite scores}

Overall, participants in each pathway experienced a significant mean increase of 3.04 (SD $=8.73$, adjusted $P<0.001)$ points in the mental composite score and 3.93 (SD $=8.73$, adjusted $P<0.001$ ) points in the physical composite score as measured by SF-36 (Table 1). The 
greatest improvement in physical composite score was observed in the orthopedic fitness pathway (mean difference $=4.83$, percent change $=11 \%$, adjusted $P<0.001$; (Table 1). Participants of the cancer fitness pathway experienced the greatest increase in mental composite score (mean difference $=3.71$, percent change $=8 \%$, adjusted $P<0.001$; Table 1$)$.

\section{Discussion}

The purpose of this investigation was to evaluate the effects of NextSteps Fitness Programs ${ }^{T M}$ on physical and health outcomes among older adults with one or more chronic conditions, including heart disease, cancer, diabetes, respiratory, obesity, and orthopedics. NextSteps Fitness Programs ${ }^{T M}$ were developed to maximize individualized exercise prescription based on participant fitness goals, recommendations from their referring health care provider, and expertise of the fitness specialist to improve functional fitness and overall quality of life.

Nearly three quarters ( $n=5,753 ; 72 \%$ ) of the participants completed the 8-week program within the six pathways. Participants generally experienced improvements in risk factors associated with chronic conditions, including BMI, body fat, perceived mental composite score, and blood pressure. The observed improvements indicate that participants have increased their overall physical function, strength, mental health, and quality of life. The magnitude of change in each of the parameters was small, albeit consistent among participants in each pathway, and was found to parallel findings from other studies on exercise referral [20-28]. An evaluation of a 20-week loaded and unloaded resistance program exhibited similar functional fitness improvements, such as a $16.94 \%$ increase in biceps curls among its participants [29]. Similarly, Hamar and colleagues [30] found a significant improvement in physical and emotional health among patients with chronic conditions following participation in the SilverSneakers ${ }^{\circledR}$ program. Despite the small changes over 8-weeks, the results may have more considerable public health significance when disseminated to a larger population.

Physical activity has shown to have a strong protective effect against colon and breast cancers [14-15,3134]. Regular physical activity among these groups was associated with up to a $50 \%$ decrease in all-cause mortality in colon cancer and $41 \%$ in breast cancer [31-34]. Recent studies have shown that regular physical activity before and after a cancer diagnosis may improve functional and physical wellbeing [14-18]. Our findings are consistent with other findings as participants within the cancer fitness pathway experienced the most significant improvement in mental composite score from pre-assessment to post-assessment $(8 \%$, adjusted $P<0.001)$ and an overall $8 \%$ increase (adjusted $P<0.001$ ) in the physical composite score.
Regular exercise is well-known to prevent cardiovascular disease such as CAD by as much as $22 \%$ in men [35] and 33\% in women [36]. Taylor and colleagues [2] described a structured exercise program as vital for patients following a cardiac event such as a Myocardial Infarction (MI), coronary intervention, or heart failure as it may lead to a reduction in mortality in this population by $20-30 \%$. Consistent with other findings, participants enrolled in the cardiac fitness pathway experienced significant improvements in estimated $\mathrm{VO}_{2}$ max, strength, and overall physical and mental health composite scores. However, despite these findings, physical activity for cardiac disease rehabilitation is infrequently utilized following hospitalization for an $\mathrm{Ml}$ or coronary intervention [37].

Participants within the pulmonary fitness pathway exhibited the most significant change in maximum oxygen uptake, as measured by $\mathrm{VO}_{2} \max (7.0 \%$, adjusted $P<0.001)$. These findings parallel the importance of physical activity in patients with COPD as $\mathrm{VO}_{2}$ max is considered reflective of disease severity [38]. The overall increase in physical (8\%) and mental (6\%) health composite scores indicate that participants perceive health benefits. Further cementing findings that exercise among patients with COPD are associated with a reduction in the forced expiratory volume $\left(F E V_{1}\right)$ decline and disease progression $[8,38]$.

Orthopedic fitness was the most popular pathway among the NextSteps Fitness Programs ${ }^{T M}$ program participants. We observed the greatest increase in the number of abdominal crunches from pre-assessment to post-assessment (mean change $=7.94$, percent change $=27.4 \%$, adjusted $P<0.001$ ) among this group serving as an indicator for improvement in muscular strength and endurance. Physical activity among this group has been shown to have a positive effect on bone mineral density and a reduction in falls and fractures among patients with osteoporosis and demonstrated reductions in pain, function, and quality of life among patients with osteoarthritis [12,13].

Participants in the diabetes fitness pathway experienced significant changes in each measured parameter, indicating that the program was effective in improving disease symptomatology. The most significant change was observed within the strength and endurance measures; however, BMI was the only parameter with the least improvement. One study found that exercise among patients with type 2 diabetes can achieve greater glycemic control and a reduced triglyceride level [39]. Although we did not measure hemoglobin A1c levels, it can be surmised that this led to greater glycemic control.

Obesity affects a considerable proportion of the American population [40]. It was not surprising that the weight management pathway had the second-highest number of participants ( $n=1,123$ ) and the only to 
experience a significant decrease in BMI (-2\%, adjusted $P<0.001)$. All participants in the weight management pathway improved their strength and endurance as well as their physical and mental health composite scores. An overall mean decrease $(-1.23 \%)$ in diastolic blood pressure along with a decreased BMI and increases in aerobic capacity, strength, and endurance observed in 8-weeks indicate that risk factors for disease progression, such as hypertension have been slightly reduced.

\section{Program model}

Next Steps Fitness Programs ${ }^{T M}$ were developed as a medically integrated, multicomponent exercise and behavioral change intervention designed to deliver individualized fitness protocols for those transitioning from or managing one or more chronic conditions. Medical integration is the program's conceptual framework, aimed at bridging the gap between fitness professionals and healthcare providers in the prevention and treatment of chronic pain and disease. Each wellness center offering Next Steps Fitness Programs ${ }^{T M}$ has a dedicated medical integration task force to oversee program delivery while utilizing non-binding recommendations from their Medical Advisory Committee, consisting of physicians, department directors, healthcare providers, rehabilitation therapists, and a resource for the wellness center.

Participants are referred to the program by a healthcare provider, either through an electronic medical record or physically bringing the referral to the wellness center. Two follow-ups are sent back to the referring provider, to close the loop on the referral and to send the pre- and post-assessment results. Upon enrollment, each participant is scheduled for 16 appointments that include a pre- and post-assessment with two 60-minute supervised exercise sessions per week for 8-weeks. An individualized exercise plan is prescribed as part of the pre-assessment consultation utilizing guidelines from the ACSM and comprised of progressive resistance training paired with functional movement training. There are on average, 8-10 participants in each session, supervised by degreed and certified fitness specialists. Each participant utilizes their exercise plan independently. Each participant begins with a 15-minute warm-up that may include brisk walking and conclude their exercise session with a 15-minute cool-down that includes upper and lower body flexibility exercises.

\section{Limitations}

The major limitation of this evaluation is the lack of a control or comparator group, which would have allowed for inferences to be made with a higher level of confidence. Moreover, the use of secondary data limited the ability to identify direct causal factors and to follow-up for disease progression assessments through the medical integration and care-provider teams. Additionally, more than half the number of participants had a follow-up assessment, which significantly decreased the sample size and limited findings among each of the cohorts as well as factors as to the reasons, such as motivation for lack of program completion. We acknowledge the Polar Ownlndex ${ }^{\circledR}$ method of estimating $\mathrm{VO}_{2}$ max has not been shown to be reliable in unhealthy populations. Lastly, although information was collected regarding the amount of time spent exercising, the lack of significant number of evaluations hindered the assessment of whether participation in NextSteps Fitness Programs $^{T M}$ enables the continuation of exercise following the 8-week program. A future evaluation of Next Steps Fitness Programs ${ }^{T M}$ will seek to mitigate these issues through a prospective, controlled study.

\section{Conclusion}

Our findings suggest that a structured and personalized fitness program, such as Next Steps, is effective and holds much promise to improve general health and slow disease progression among older adults with one or more chronic conditions. Participants of the six chronic disease pathways showed improvements in various key health measures. The present study serves as a model for reproducibility to implement medically integrated fitness and enhance physician-led exercise prescription in chronic conditions and providing insight into the realized benefits of supervised structured exercise adapted and implemented for specialized populations. across 11 states, it also demonstrates that a structured and personalized fitness program can be replicated and effective in diverse populations. The findings from this evaluation will serve as a model for reproducibility to implement medically integrated fitness and enhance physician-led exercise prescription in chronic conditions and provide insight into the realized benefits of supervised structured exercise adapted and implemented for specialized populations.

\section{Conflict of Interest}

The authors declare no potential conflict of interest with respect to the research, authorship, and/or publication of this article.

\section{Acknowledgements}

The results of this study do not constitute endorsement by the American College of Sports Medicine. The authors hereby declare the results of the study are presented clearly, honest, and without fabrication, falsification, or inappropriate data manipulation.

\section{References}

1. Kochanek KD, Murphy SL, Xu J, Arias E (2017) Mortality in the United States, 2016. National Center for Health Statistics Data Brief 293: 1-8.

2. Taylor RS, Unal B, Critchley JA, Capewell S (2006) Mortality reductions in patients receiving exercise-based cardiac rehabilitation: how much can be attributed to cardiovascular risk factor improvements? Eur J Cardiovasc PrevRehabil 13: $369-374$ 
3. Ortman JM, Velkoff VA, Hogan H (2014) An aging nation: The older population in the United states. Current Population Reports, U.S. Census Bureau.

4. Brach JS, Simonsick EM, Kritchevsky S, Yaffe K, Newman AB (2004) The association between physical function and lifestyle activity and exercise in the health, aging and body composition study. J Am Geriatr Soc 52: 502-509.

5. Lee PG, Jackson EA, Richardson CR (2017) Exercise prescriptions in older adults. Am Fam Physician 95: 425-432.

6. Blair SN, Kampert JB, Kohl HW 3rd, Barlow CE, Macera CA, et al. (1996) Influences of cardio respiratory fitness and other precursors on cardiovascular disease and all-cause mortality in men and women. JAMA 276: 205-210.

7. Manson JE, Greenland P, LaCroix AZ, Stefanick ML, Mouton $C P$, et al. (2002) Walking compared with vigorous exercise for the prevention of cardiovascular events in women. N Engl J Med 347:716-725.

8. McGlone S, Venn A, Walters EH, Wood-Baker R (2006) Physical activity, spirometry and quality-of-life in chronic obstructive pulmonary disease. COPD 3: 83-88.

9. Mead GE, Morley W, Campbell P, Greig CA, McMurdo M, et al. (2009) Exercise for depression. Cochrane Database of Systematic Reviews Issue 3. Art. No.: CD004366.

10. Kuk JL, Davachi S, Kriska AM, Riddell M, Gregg EW (2010) Pre-diabetes detection and intervention for high risk communities. J Phys Act Health 7: 327-340.

11. Rogers LQ, Madera CA, Hootman JM, Ainsworth BE, Blair SN (2002) The association between joint stress from physical activity and self-reported osteoarthritis: an analysis of the Cooper Clinic data. Osteoarthritis Cartilage 10: 617-622.

12. Chillibeck PD, Sale DG, Webber CE (1995) Exercise and bone mineral density. Sports Med 19: 103-122.

13. Kohrt WM, Bloomfield SA, Little KD, Nelson ME, Yingling VR (2004) American College of Sports Medicine. American college of Sports Medicine position stand: physical activity and bone health. Med Sci Sports Exerc 36: 1985-1996.

14. Nilsen TI, Romundstad PR, Petersen H, Gunnell D, Vatten LJ (2008) Recreational physical activity and cancer risk in subsites of the colon. Nord-Trøndelag Health Study. Cancer Epidemiol Biomarkers Prev 17: 183-188.

15. Bernstein L, Patel AV, Ursin G, Sullivan-Halley J, Press MF, et al. (2005) Lifetime recreational exercise activity and breast cancer risk among black women and white women. $J$ Natl Cancer Inst 97: 1671-1679.

16. Furberg AS, Thune I (2003) Metabolic abnormalities (hypertension, hyperglycemia and overweight), lifestyle (high energy intake and physical inactivity) and endometrial cancer risk in a Norwegian cohort. Int J Cancer 104: 669-676.

17. Friedenreich CM, Thune I (2001) A review of physical activity and prostate cancer. Cancer Causes Control 12: 461-475.

18. U.S. Department of Health and Human Services (2018) Physical activity guidelines for Americans. 2nd Edition.

19. Pucci GC, Rech CR, Fermino RC, Reis RS (2012) Association between physical activity and quality of life in adults. Rev Saude Publica 46: 166-179.

20. Pavey TG, Taylor AH, Fox KR, Hillsdon M, Anokye N, et al. (2011) Effect of exercise referral schemes in primary care on physical activity and improving health outcomes: systematic review and meta-analysis. BMJ 4: 6462.

21. Pavey TG, Anokye N, Taylor AH, Trueman P, Moxham T, et al. (2011) The clinical effectiveness and cost-effectiveness of exercise referral schemes: a systematic review and economic evaluation. Health Technol Assess 15: 1-254.

22. Williams $\mathrm{NH}$, Hendry M, France $B$, Lewis R, Wilkson $C$ (2007) Effectiveness of exercise referral schemes to promote physical activity in adults: systematic review. Br J Gen Pract 57: 979-986.
23. Benjamin EJ, Blaha MJ, Chiuve SE, Cushman M, Das SR, et al. (2017) Heart disease and stroke statistics-2017 update: A report from the American Heart Association. Circulation 135: 1-458.

24. Costello E, Leone JE, Ellzy M, Miller TA (2013) Older adult perceptions of the physicians' role in promoting physical activity. Disabil Rehabil 35: 1191-1198.

25. Zaleski AL, Taylor BA, Panza GA, Wu Y, Pescatello LS, et al. (2016) Coming of age: Considerations in the prescription of exercise for older adults. Methodist Debakey Cardiovasc J 12: 98-104.

26. Pescatello L, Arena R, Riebe D, Thompson P (2013) General principles of exercise prescription. In ACSM's guidelines for exercise testing and prescription. Philadelphia: Wolters Kluwer Health/Lippincott Williams \& Wilkins 166-77.

27. Sousa N, Mendes R, Abrantes C, Sampaio J, Oliveira J (2014) Effectiveness of combined exercise training to improve functional fitness in older adults: A randomized controlled trial. Geriatr Gerontollnt 14: 892-898.

28. Kang S, Hwang S, Klein AB, Kim SH (2015) Multicomponent exercise for physical fitness of community-dwelling elderly women. J Phys Ther Sci 27: 911-915.

29. Glenn JM, Gray M, Binns A (2015) The effects of loaded and unloaded high-velocity resistance training on functional fitness among community-dwelling older adults. Age Ageing 44: 926-931.

30. Hamar B, Coberley CR, Pope JE, Rula EY (2013) Impact of a senior fitness program on measures of physical and emotional health and functioning. Popul Health Manag 16: 364-372.

31. Ibrahim E, Al-Homaidh A (2011) Physical activity and survival after breast cancer diagnosis: meta-analysis of published studies. Med Oncol 28: 753-765.

32. Patterson RE, Cadmus LA, Emond JA, Pierce JP (2010) Physical activity, diet, adiposity and female breast cancer prognosis: A review of the epidemiologic literature. Maturitas 66: 5-15.

33. Meyerhardt JA, Giovannucci EL, Holmes MD, Chan AT, Chan JA, et al. (2006) Physical activity and survival after colorectal cancer diagnosis. J Clin Oncol 24: 3527-3534.

34. Meyerhardt, JA, Heseltine D, Niedzwiecki D, Hollis D, Saltz LB, et al. (2006) Impact of physical activity on cancer recurrence and survival in patients with stage III colon cancer: findings from CALGB 89803. J Clin Oncol 24: 3535-3541.

35. Sattelmair J, Pertman J, Ding E, Kohl H, Haskell W, et al. (2011) Dose response between physical activity and risk of coronary heart disease: A meta-analysis. Circulation 124: 789-795.

36. Manson JE, Hu FB, Rich-Edwards JW, Colditz GA, Stampfer MJ, et al. (1999) A prospective study of walking as compared with vigorous exercise in the prevention of coronary heart disease in women. N Engl J Med 341: 650-658.

37. Suaya JA, Shepard DS, Normand SL, Ades PA, Prottas $\mathrm{J}$, et al. (2007) Use of cardiac rehabilitation by Medicare beneficiaries after myocardial infarction or coronary bypass surgery. Circulation 116: 1653-1662.

38. Ganju AA, Fuladi AB, Tayade BO, Ganju NA (2011) Cardiopulmonary exercise testing in evaluation of patients of chronic obstructive pulmonary disease. Indian J Chest Dis Allied Sci 53: 87-91.

39. Thomas D, Elliot EJ, Naughton GA (2006) Exercise for type 2 diabetes mellitus. Cochrane Database Syst Rev 19: CD002968.

40. Hales CM, Carroll MD, Fryar CD, Ogden CL (2017) Prevalence of obesity among adults and youth: United States, 2015-2016. NCHS Data Brief 1-8. 